\title{
Apoptotic cell clearance lets you breathe easy
}

The epithelial cell barrier is emerging as an important modulator of tolerance and immunity at mucosal sites. Reporting in Nature, Ravichandran and colleagues now show that airway epithelial cells can engulf apoptotic epithelial cells and establish an anti-inflammatory environment that limits allergic airway inflammation.

Airway epithelial cells can undergo apoptosis following exposure to environmental pollutants, allergens and pathogens. Indeed, aggregates of uncleared epithelial cell corpses (termed Creola bodies) have long been described in the sputum of asthmatics. Because epithelial cells greatly outnumber professional phagocytes at this site, the authors investigated whether epithelial cells can clear apoptotic cells from the airways. Indeed, human and mouse epithelial cell lines, as well as freshly isolated mouse airway epithelial cells, were shown to engulf apoptotic cells and produce anti-inflammatory cytokines in vitro. This process was dependent on phosphatidylserine exposure on the apoptotic cells and on epithelial cell expression of the small GTPase RAC1, which functions downstream of several engulfment receptors.

To assess the in vivo role of engulfment by airway epithelial cells, the authors developed a mouse model (CCSP-Cre/Rac1 $1^{f l f l}$ mice) in which RAC1 expression can be inducibly deleted specifically in airway epithelial cells. Following RAC1 deletion, these mice were primed and challenged with house dust mite (HDM) allergen that has low endotoxin content through the intranasal route. These mice developed severe airway inflammation, which was characterized by high levels of the $\mathrm{T}$ helper $2\left(\mathrm{~T}_{\mathrm{H}} 2\right)$-type cytokines interleukin-4 (IL-4), IL-5 and IL-13,

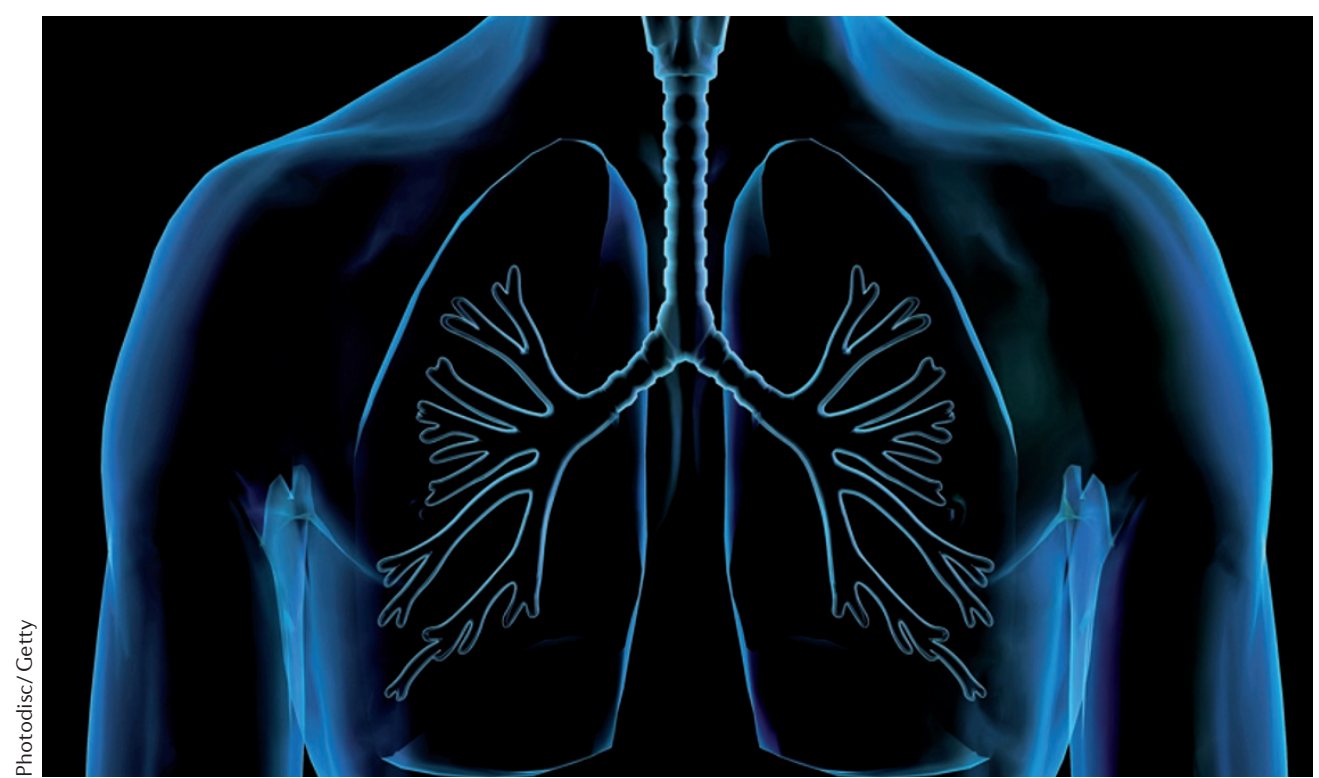

immune cell recruitment to the lungs and other features of airway hyperresponsiveness. By contrast, control mice showed minimal airway inflammation following allergen exposure. Interestingly, exposure of RAC1-sufficient mice to allergen resulted in high IL-10 levels in the lungs, whereas IL-10 levels were not increased in CCSP-Cre/Rac1 $1^{f l f l}$ mice. Furthermore, administration of recombinant IL-10 to CCSP-Cre/ $R a c 1^{f l f l}$ mice during the allergen priming and challenge phases rescued the airway inflammatory phenotype. Similar results were observed when ovalbumin (without adjuvant) was used as the allergen.

High levels of IL-33 and high numbers of IL- $5^{+}$group 2 innate lymphoid cells (ILCs; also known as nuocytes) were also observed in the lungs of CCSP-Cre/Racl ${ }^{f l / f l}$ mice after allergen challenge. Neutralization of IL-33 in these mice reduced the allergic inflammatory response, and further analysis showed that RAC1deficient epithelial cells were the main source of this IL-33.

Together, this study shows that engulfment of dead cells by lung epithelial cells and increased IL-10 production helps to prevent airway inflammation in response to normally innocuous inhaled allergens. Disruption of engulfment by airway epithelial cells results in increased IL-33 production, increased numbers of lung IL- $5^{+}$ILCs and allergic inflammation.

Olive Leavy

ORIGINAL RESEARCH PAPER Juncadella, I. J. et al. Apoptotic cell clearance by bronchial epithelial cells critically influences airway inflammation. Nature 493, 547-551 (2013) 